03

\title{
Решение задачи дифракции на теле вращения, расположенном в диэлектрическом слое
}

\author{
(C) C.A. Маненков \\ Московский технический университет связи и информатики, \\ 111024 Москва, Россия \\ e-mail: mail44471@mail.ru
}

Поступила в редакцию 10.06.2019 г.

В окончательной редакции 02.08.2019 г.

Принята к публикации 12.08.2019 г.

\begin{abstract}
Предложены два метода решения задачи дифракции плоской волны на диэлектрическом теле вращения, расположенном в однородном диэлектрическом слое. Проведено сравнение двух методов на примере задачи рассеяния плоской волны на однородном сфероиде и круговом цилиндре конечных размеров, расположенных в слое. Представлены результаты расчета диаграммы рассеяния для случая дифракции на конечном круговом цилиндре, который погружен в слой и имеет переменные материальные характеристики. Получены зависимости диаграммы рассеяния от длины волны для плазмонных частиц, расположенных в слоистой среде.
\end{abstract}

Ключевые слова: рассеяние волн в плоскослоистых средах, метод дискретных источников, метод интегральных уравнений.

DOI: $10.21883 /$ OS.2019.12.48690.214-19

\section{1. Введение}

Задача дифракции волн на компактных препятствиях, расположенных в неоднородных средах, представляет большой практический интерес. В частности, в литературе рассматривались различные двумерные задачи дифракции электромагнитных волн [1-3], а также трехмерные задачи дифракции [4-6] на телах, расположенных в плоскослоистых средах. В настоящей работе исследована задача рассеяния плоской волны на диэлектрическом теле вращения, расположенном в однородном диэлектрическом слое. Предполагается, что ось вращения тела перпендикулярна границе раздела сред. В работе рассмотрены два метода решения указанной задачи дифракции: модифицированный метод дискретных источников (ММДИ) [7,8] и модифицированный метод объемного интегрального уравнения (ММОИУ) [9]. Отметим, что ММОИУ является приближенным подходом. Этот метод близок к методу продолженных граничных условий (МПГУ), который ранее успешно применялся к решению различных задач дифракции [10].

В случае применения ММДИ предполагается, что материальные характеристики среды внутри рассеивателя постоянны, а при использовании ММОИУ считаем, что диэлектрическая проницаемость тела является функцией координат, не зависящей от угловой координаты (в цилиндрической системе координат, связанной с телом вращения). В обоих случаях для решения задачи использовалась матричная функция Грина $(Ф Г)$, учитывающая слоистый характер среды. Такой подход позволяет свести задачу к системе интегральных уравнений относительно некоторых функций, распределенных на вспомогательных поверхностях, расположенных внутри и вне исходной поверхности тела (при использовании ММДИ) или распределенных внутри области, занимаемой рассеивателем (при использовании ММОИУ). При этом отпадает необходимость рассматривать неизвестное поле на границе раздела сред.

Отметим, что данная работа является обобщением работ $[9,11]$ на случай дифракции на теле вращения, расположенном в слое. Для ускорения вычислительных алгоритмов, основанных на ММДИ и ММОИУ, ядро интегрального уравнения (или системы уравнений), к которому сводится задача дифракции, разбивали на сингулярную и регулярную части. В случае применения ММОИУ сингулярную часть ядра заменяли близким ядром, не имеющим особенности при совпадении аргументов. Алгебраизация задачи (в обоих методах) осуществлялась при помощи разложения ядер и неизвестных функций в ряд Фурье. При использовании ММДИ соответствующие одномерные интегральные уравнения решались методом коллокации. В случае применения ММОИУ исходное объемное интегральное уравнение сводилось к системе двумерных уравнений по осевому сечению тела. Последние решали при помощи метода Крылова-Боголюбова. При этом матричные элементы алгебраических систем также разбивали на два слагаемых, обусловленных сингулярной и регулярной частями ядер интегральных уравнений. Так как регулярные части ядер интегральных уравнений являются медленно меняющимися функциями координат, то интегралы, через которые выражаются соответствующие части матричных элементов, можно вычислять, используя, например, 
квадратуры невысокого порядка. Это позволило существенно сократить объем требуемых вычислений.

\section{2. Решение задачи дифракции на основе ММдИ}

Рассмотрим постановку задачи. Пусть компактный рассеиватель в виде тела вращения расположен внутри диэлектрического слоя с характеристиками $\varepsilon_{2}, \mu_{2}$. Диэлектрическую и магнитную проницаемости среды, окружающей слой, обозначим через $\varepsilon_{1}$ и $\mu_{1}$. Характеристики среды внутри тела $-\varepsilon_{i}, \mu_{i}$. Предполагаем, что ось симметрии рассеивателя перпендикулярна границам диэлектрического слоя. Введем декартову систему координат, причем ось $z$ направим вдоль оси симметрии рассеивателя (рис. 1). Выберем начало системы координат внутри области $D$, занимаемой телом. Обозначим через $S$ границу области $D$. В указанной системе координат границы диэлектрического слоя имеют вид $z=-d_{1}$ и $z=d_{2}$. Предполагаем, что на границе $S$ рассеивателя выполнены условия сопряжения:

$$
\left.[\mathbf{n} \times \mathbf{E}]\right|_{S}=0,\left.\quad[\mathbf{n} \times \mathbf{H}]\right|_{S}=0 .
$$

Здесь $\mathbf{n}-$ нормаль, внешняя к поверхности тела, $\mathbf{E}, \mathbf{H}-$ напряженности полных электрического и магнитного полей, квадратные скобки означают скачок соответствующей величины. На границах раздела сред $z=-d_{1}$ и $z=d_{2}$ выполнены условия сопряжения вида:

$$
\begin{gathered}
{\left.\left[\mathbf{i}_{z} \times \mathbf{E}\right]\right|_{z=-d_{1}}=0,\left.\quad\left[\mathbf{i}_{z} \times \mathbf{H}\right]\right|_{z=-d_{1}}=0,} \\
{\left.\left[\mathbf{i}_{z} \times \mathbf{E}\right]\right|_{z=d_{2}}=0,\left.\quad\left[\mathbf{i}_{z} \times \mathbf{H}\right]\right|_{z=d_{2}}=0,}
\end{gathered}
$$

где $\mathbf{i}_{z}$ - единичный орт вдоль оси $z$.

Предполагаем, что структура облучается плоской волной, падающей из нижнего полупространства $\left(z<-d_{1}\right)$, причем будем рассматривать две поляризации падающего поля. В первом случае

$$
\begin{gathered}
E_{y}^{i}=\exp \left(-i k_{1} x \sin \chi_{1}-i k_{1} z \cos \chi_{1}\right), \\
E_{x}^{i}=E_{z}^{i}=0
\end{gathered}
$$

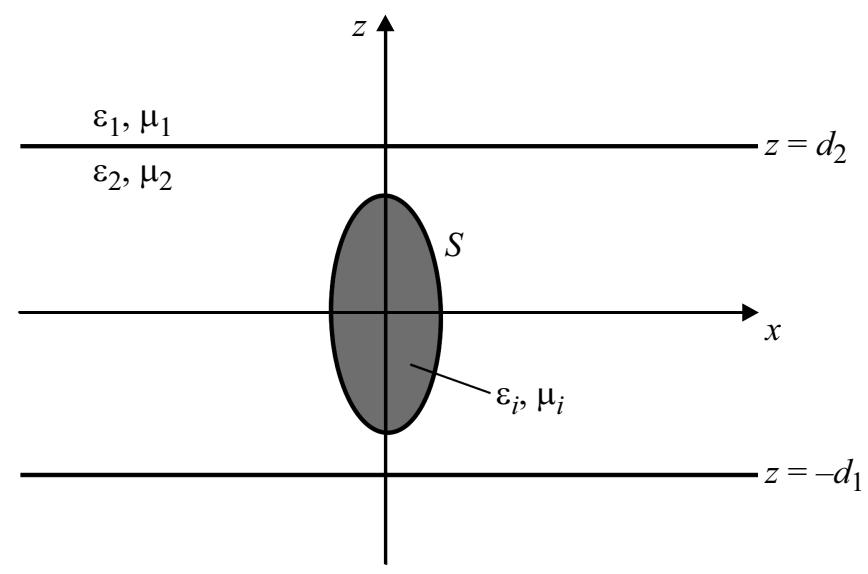

Рис. 1. Геометрия задачи. а во втором

$$
\begin{gathered}
H_{y}^{i}=\exp \left(-i k_{1} x \sin \chi_{1}-i k_{1} z \cos \chi_{1}\right), \\
H_{x}^{i}=H_{z}^{i}=0,
\end{gathered}
$$

где $\chi_{1}-$ угол падения плоской волны, $k_{1}=\omega \sqrt{\varepsilon_{1} \mu_{1}}-$ волновое число среды вне слоя, $\omega-$ круговая частота. Остальные компоненты падающего поля определяются из уравнений Максвелла. Введем также в рассмотрение первичное и вторичное (рассеянное) поля. А именно

$$
\mathbf{E}=\mathbf{E}^{0}+\mathbf{E}^{1}, \quad \mathbf{H}=\mathbf{H}^{0}+\mathbf{H}^{1},
$$

где напряженности первичного поля $\mathbf{E}^{0}, \mathbf{H}^{0}$ определяются из задачи дифракции плоской волны (3) или (4) на диэлектрическом слое в отсутствие компактного тела. Решение данной задачи общеизвестно и здесь не приводится. Будем также считать, что на бесконечности выполнены условия излучения для рассеянного поля:

$$
\begin{gathered}
\lim _{r \rightarrow \infty} \mathbf{E}^{1}=0, \quad \lim _{r \rightarrow \infty} \mathbf{H}^{1}=0, \\
\operatorname{Im} \varepsilon_{p}, \mu_{p}<0, \quad p=1,2,
\end{gathered}
$$

где $r$ - расстояние от начала координат до точки наблюдения. При этом в соответствии с принципом предельного поглощения вначале предполагаем, что проницаемости всех сред имеют малые мнимые части, а затем в окончательных формулах устремляем мнимые части к нулю.

Будем решать задачу при помощи ММДИ. Представим поле внутри и вне тела в виде

$$
\begin{gathered}
\mathbf{E}(\mathbf{r})= \begin{cases}\int_{\Sigma_{2}} \mathbf{G}_{2}^{E}\left(\mathbf{r}, \mathbf{r}^{\prime}\right) \mathbf{J}_{2}\left(\mathbf{r}^{\prime}\right) d s^{\prime}, & \mathbf{r} \in D, \\
\mathbf{E}^{0}(\mathbf{r})+\int_{\Sigma_{1}} \mathbf{G}_{1}^{E}\left(\mathbf{r}, \mathbf{r}^{\prime}\right) \mathbf{J}_{1}\left(\mathbf{r}^{\prime}\right) d s^{\prime}, & \mathbf{r} \in \mathbf{R}^{3} \backslash D,\end{cases} \\
\mathbf{H}(\mathbf{r})= \begin{cases}\int_{\Sigma_{2}} \mathbf{G}_{2}^{H}\left(\mathbf{r}, \mathbf{r}^{\prime}\right) \mathbf{J}_{2}\left(\mathbf{r}^{\prime}\right) d s^{\prime}, & \mathbf{r} \in D, \\
\mathbf{H}^{0}(\mathbf{r})+\int_{\Sigma_{1}} \mathbf{G}_{1}^{H}\left(\mathbf{r}, \mathbf{r}^{\prime}\right) \mathbf{J}_{1}\left(\mathbf{r}^{\prime}\right) d s^{\prime}, & \mathbf{r} \in \mathbf{R}^{3} \backslash D,\end{cases}
\end{gathered}
$$

где $\mathbf{G}_{1}^{E, H}$ и $\mathbf{G}_{2}^{E, H}$ - матричные ФГ слоистой среды вне рассеивателя и среды внутри тела, $\Sigma_{1}$ и $\Sigma_{2}-$ вспомогательные поверхности вращения, расположенные внутри и вне исходной поверхности тела $S$, а $\mathbf{J}_{1}$ и $\mathbf{J}_{2}-$ неизвестные токи, распределенные на поверхностях $\Sigma_{1}$ и $\Sigma_{2}$. Заметим, что выбор вспомогательных поверхностей $\Sigma_{1}$ и $\Sigma_{2}$ осуществляется при помощи аналитической деформации исходной границы рассеивателя, как это описано в работах $[11,12]$. В дальнейшем будем считать, что поверхность тела $S$ и вспомогательные поверхности $\Sigma_{1}$ и $\Sigma_{2}$ заданы в подходящей ортогональной системе координат $(\alpha, \beta, \varphi)$ уравнениями $\alpha=\alpha(\beta)$ и $\alpha=\alpha_{\Sigma_{p}}\left(\beta^{\prime}\right)$, $\beta=\beta_{\Sigma_{p}}\left(\beta^{\prime}\right)(p=1,2)$, где $\beta^{\prime}$ - параметр, изменяющийся в интервале $[0, \pi][11,12]$. 
Матричные $Ф Г \mathbf{G}_{2}^{E, H}$ внутри тела определяются в соответствии с формулами $[11,12]$

$$
\begin{gathered}
\mathbf{G}_{2}^{E}\left(\mathbf{r}, \mathbf{r}^{\prime}\right) \mathbf{J}_{2}\left(\mathbf{r}^{\prime}\right)=\mathbf{A}_{i}+\frac{1}{k_{i}^{2}} \nabla\left(\nabla \cdot \mathbf{A}_{i}\right), \\
\mathbf{G}_{2}^{H}\left(\mathbf{r}, \mathbf{r}^{\prime}\right) \mathbf{J}_{2}\left(\mathbf{r}^{\prime}\right)=\frac{i}{k_{i} \xi_{i}} \nabla \times \mathbf{A}_{i},
\end{gathered}
$$

где

$$
\mathbf{A}_{i}=\mathbf{J}_{2}\left(\mathbf{r}^{\prime}\right) G_{i}\left(\mathbf{r}, \mathbf{r}^{\prime}\right), \quad G_{i}=\frac{\exp \left(-i k_{i} R\right)}{4 \pi R}, \quad R=\left|\mathbf{r}-\mathbf{r}^{\prime}\right| .
$$

В формулах (9)-(11) $k_{i} \omega \sqrt{\varepsilon_{i} \mu_{i}}$ - волновое число среды внутри тела, $\xi_{i}=\sqrt{\mu_{i} / \varepsilon_{i}}-$ волновой импеданс среды тела. Для построения ФГ слоистой среды, в которой расположено тело, т.е. матричных функций $\mathbf{G}_{1}^{E}$ и $\mathbf{G}_{1}^{H}$, применим методику, описанную в работе [13]. Для вывода представлений этих величин рассматривалась вспомогательная задача. Обозначим

$$
\begin{aligned}
\mathbf{E}^{G}\left(\mathbf{r}, \mathbf{r}^{\prime}\right) & =\mathbf{G}_{1}^{E}\left(\mathbf{r}, \mathbf{r}^{\prime}\right) \mathbf{J}_{1}\left(\mathbf{r}^{\prime}\right), \\
\mathbf{H}^{G}\left(\mathbf{r}, \mathbf{r}^{\prime}\right) & =\mathbf{G}_{1}^{H}\left(\mathbf{r}, \mathbf{r}^{\prime}\right) \mathbf{J}_{1}\left(\mathbf{r}^{\prime}\right) .
\end{aligned}
$$

Поля $\mathbf{E}^{G}$ и $\mathbf{H}^{G}$ удовлетворяют уравнениям Максвелла:

$$
\begin{gathered}
\nabla \times \mathbf{E}^{G}=-i k_{2} \xi_{2} \mathbf{H}^{G}, \\
\nabla \times \mathbf{H}^{G}=\frac{i k_{2}}{\xi_{2}} \mathbf{E}^{G}+\frac{i}{k_{2} \xi_{2}} \mathbf{J}_{1}\left(\mathbf{r}^{\prime}\right) \delta\left(\mathbf{r}-\mathbf{r}^{\prime}\right), \quad-d_{1} z<d_{2} \\
\nabla \times \mathbf{E}^{G}=-i k_{1} \xi_{1} \mathbf{H}^{G}, \quad \nabla \times \mathbf{H}^{G}=\frac{i k_{1}}{\xi_{1}} \mathbf{E}^{G} \\
z<-d_{1}, \quad z>d_{2},
\end{gathered}
$$

условиям сопряжения вида (2) на границе раздела и условиям (6) на бесконечности. В (14) и (15) $k_{2}=\omega \sqrt{\varepsilon_{2} \mu_{2}}-$ волновое число среды слоя, $\xi_{2}=\sqrt{\mu_{2} / \varepsilon_{2}}$ и $\xi_{1}=\sqrt{\mu_{1} / \varepsilon_{1}}-$ волновые импедансы среды слоя и окружающего слой пространства. В дальнейшем перейдем к цилиндрическим координатам $(\rho, \varphi, z)$. Тогда для нахождения компонент матричных $Ф Г \mathbf{G}_{1}^{E}$ и $\mathbf{G}_{1}^{H}$ необходимо рассмотреть три задачи, соответствующие возбуждению слоистой среды сторонними токами следующего вида:

$$
\mathbf{J}_{1}=\mathbf{i}_{\rho^{\prime}}(\mathrm{I}), \quad \mathbf{J}_{1}=\mathbf{i}_{\varphi^{\prime}}(\mathrm{II}), \quad \mathbf{J}_{1}=\mathbf{i}_{z^{\prime}}(\mathrm{III}),
$$

где $\left(\rho^{\prime}, \varphi^{\prime}, z^{\prime}\right)$ - цилиндрические координаты точки, в которой расположен точечный источник, а $\mathbf{i}_{\rho^{\prime}}, \mathbf{i}_{\varphi^{\prime}}, \mathbf{i}_{z^{\prime}}$ единичные орты по осям координат в точке источника. При этом координаты векторов $\mathbf{E}^{G}$ и $\mathbf{H}^{G}$, полученные при решении задач (I)-(III), образуют столбцы матричных ФГ $\mathbf{G}_{1}^{E}$ и $\mathbf{G}_{1}^{H}$.

Во всех трех случаях удобно представить функции $\mathbf{E}^{G}$ и $\mathbf{H}^{G}$ в виде

$$
\mathbf{E}^{G}=\mathbf{A}+\frac{1}{k^{2}(z)} \nabla(\nabla \cdot \mathbf{A}),
$$

$$
\mathbf{H}^{G}=\frac{i}{k(z) \xi(z)} \nabla \times \mathbf{A},
$$

где

$$
\begin{aligned}
& k(z)= \begin{cases}k_{1}, & z<-d_{1}, z>d_{2}, \\
k_{2}, & -d_{1}<z<d_{2},\end{cases} \\
& \zeta(z)= \begin{cases}\xi_{1}, & z_{1}<-d_{1}, z>d_{2}, \\
\xi_{2}, & -d_{1}<z<d_{2} .\end{cases}
\end{aligned}
$$

В формулах (17) и (18) потенциал А определяется из соотношения (в декартовых координатах) [13]

$$
\mathbf{A}=\left(\begin{array}{ccc}
f_{11} & 0 & 0 \\
0 & f_{11} & 0 \\
\frac{\partial f_{31}}{\partial x} & \frac{\partial f_{31}}{\partial y} & f_{33}
\end{array}\right) \mathbf{J}_{1} .
$$

Функции $f_{11}, f_{31}, f_{33}$ представляются в виде

$$
\begin{aligned}
& f_{11}=\frac{1}{2 \pi} \int_{0}^{\infty} v_{11}\left(\kappa, z, z^{\prime}\right) J_{0}\left(\kappa R_{\perp}\right) \kappa d \kappa, \\
& f_{31}=\frac{1}{2 \pi} \int_{0}^{\infty} v_{31}\left(\kappa, z, z^{\prime}\right) J_{0}\left(\kappa R_{\perp}\right) \kappa d \kappa, \\
& f_{33}=\frac{1}{2 \pi} \int_{0}^{\infty} v_{33}\left(\kappa, z, z^{\prime}\right) J_{0}\left(\kappa R_{\perp}\right) \kappa d \kappa,
\end{aligned}
$$

где $R_{\perp}=\sqrt{\left(x-x^{\prime}\right)^{2}+\left(y-y^{\prime}\right)^{2}}$, причем спектральные функции $v_{11}, v_{31}, v_{33}$ являются решениями одномерных краевых задач [13]:

$$
\begin{aligned}
& \left\{\begin{array}{l}
v_{11}^{\prime}+\left(k^{2}(z)-\kappa^{2}\right) v_{11}=-\delta\left(z-z^{\prime}\right), \\
{\left.\left[v_{11}\right]\right|_{z=-d_{1}}=0,\left.\quad\left[v_{11}\right]\right|_{z=d_{2}}=0,} \\
{\left.\left[\frac{1}{\mu} v_{11}^{\prime}\right]\right|_{z=-d_{1}}=0,\left.\quad\left[\frac{1}{\mu} v_{11}^{\prime}\right]\right|_{z=d_{2}}=0,}
\end{array}\right. \\
& \left\{\begin{array}{l}
v_{31}^{\prime \prime}+\left(k^{2}(z)-\kappa^{2}\right) v_{31}=0, \\
{\left.\left[\frac{1}{\mu} v_{31}\right]\right|_{z=-d_{1}}=0,\left.\quad\left[\frac{1}{\mu} v_{31}\right]\right|_{z=d_{2}}=0,} \\
{\left.\left[\frac{1}{\mu \varepsilon} v_{31}^{\prime}\right]\right|_{z=-d_{1}}=-\left.v_{11}\left[\frac{1}{\mu \varepsilon}\right]\right|_{z=-d_{1}},} \\
{\left.\left[\frac{1}{\mu \varepsilon} v_{31}^{\prime}\right]\right|_{z=d_{2}}=-\left.v_{11}\left[\frac{1}{\mu \varepsilon}\right]\right|_{z=d_{2}},}
\end{array}\right. \\
& \left\{\begin{array}{l}
v_{33}^{\prime \prime}+\left(k^{2}(z)-\kappa^{2}\right) v_{33}=-\delta\left(z-z^{\prime}\right), \\
{\left.\left[\frac{1}{\mu} v_{33}\right]\right|_{z=-d_{1}}=0,\left.\quad\left[\frac{1}{\mu} v_{33}\right]\right|_{z=d_{2}}=0,} \\
{\left.\left[\frac{1}{\mu \varepsilon} v_{33}^{\prime}\right]\right|_{z=-d_{1}}=0,\left.\quad\left[\frac{1}{\mu \varepsilon} v_{33}^{\prime}\right]\right|_{z=d_{2}}=0 .}
\end{array}\right.
\end{aligned}
$$

В формулах (24)-(26) функции $\varepsilon(z)$ и $\mu(z)$ определяются аналогично соотношениям (19), штрих означает 
производную по переменной $z$. Решения задач (24)(26) находятся аналогично тому, как это описано в работе [13]. Далее нетрудно показать, что в цилиндрических координатах потенциал А выражается следующим образом:

$$
\begin{gathered}
A_{\rho}=f_{11} \cos \left(\varphi-\varphi^{\prime}\right), \\
A_{\varphi}=-f_{11} \sin \left(\varphi-\varphi^{\prime}\right), \\
A_{z}=-\frac{\partial f_{31}}{\partial \rho^{\prime}}, \\
A_{\rho}=f_{11} \sin \left(\varphi-\varphi^{\prime}\right), \\
A_{\varphi}=f_{11} \cos \left(\varphi-\varphi^{\prime}\right), \\
A_{z}=-\frac{1}{\rho^{\prime}} \frac{\partial f_{31}}{\partial \varphi^{\prime}}, \\
A_{\rho}=0, \quad A_{\varphi}=0, \quad A_{z}=f_{33}
\end{gathered}
$$

соответственно для задач (I), (II) и (III).

Перейдем к решению поставленной задачи дифракции. Для этого подставим формулы (7) и (8) в граничные условия (1). В результате получим следующую систему интегральных уравнений:

$$
\begin{gathered}
\mathbf{n} \times \int_{\Sigma_{1}} \mathbf{G}_{1}^{E}\left(\mathbf{r}, \mathbf{r}^{\prime}\right) \mathbf{J}_{1}\left(\mathbf{r}^{\prime}\right) d s^{\prime} \\
\quad-\mathbf{n} \times \int_{\Sigma_{2}} \mathbf{G}_{2}^{E}\left(\mathbf{r}, \mathbf{r}^{\prime}\right) \mathbf{J}_{2}\left(\mathbf{r}^{\prime}\right) d s^{\prime}=-\mathbf{n} \times \mathbf{E}^{0}, \\
\mathbf{n} \times \int_{\Sigma_{1}} \mathbf{G}_{1}^{H}\left(\mathbf{r}, \mathbf{r}^{\prime}\right) \mathbf{J}_{1}\left(\mathbf{r}^{\prime}\right) d s^{\prime} \\
-\mathbf{n} \times \int_{\Sigma_{2}} \mathbf{G}_{2}^{H}\left(\mathbf{r}, \mathbf{r}^{\prime}\right) \mathbf{J}_{2}\left(\mathbf{r}^{\prime}\right) d s^{\prime}=-\mathbf{n} \times \mathbf{H}^{0}, \quad \mathbf{r} \in S .
\end{gathered}
$$

Для алгебраизации задачи разложим неизвестные функции (токи) и ФГ в ряды Фурье:

$$
\begin{gathered}
\mathbf{J}_{p}\left(\rho^{\prime}, z^{\prime}, \varphi^{\prime}\right)=\sum_{m=-\infty}^{\infty} \mathbf{J}_{p, m}\left(\rho^{\prime}, z^{\prime}\right) \exp \left(i m \varphi^{\prime}\right) \\
\mathbf{G}_{p}^{E}\left(\rho, z, \rho^{\prime}, z^{\prime}, \varphi-\varphi^{\prime}\right)= \\
=\sum_{m=-\infty}^{\infty} \mathbf{G}_{p, m}^{E}\left(\rho, z, \rho^{\prime}, z^{\prime}\right) \exp \left(i m\left(\varphi-\varphi^{\prime}\right)\right) \\
\mathbf{G}_{p}^{H}\left(\rho, z, \rho^{\prime}, z^{\prime}, \varphi-\varphi^{\prime}\right)= \\
=\sum_{m=-\infty}^{\infty} \mathbf{G}_{p, m}^{H}\left(\rho, z, \rho^{\prime}, z^{\prime}\right) \exp \left(i m\left(\varphi-\varphi^{\prime}\right)\right), \quad p=1,2 .
\end{gathered}
$$

Сделаем замену $[11,12]$

$$
\mathbf{J}_{p, m}=\mathbf{I}_{p}^{m} /\left(h_{\varphi} h_{\Sigma_{p}} \sqrt{\dot{\alpha}_{\Sigma_{p}}^{2}+\dot{\beta}_{\Sigma_{p}}^{2}}\right),
$$

где

$$
\mathbf{I}_{p}^{m}=-n_{\beta_{p}} I_{p}^{m 1} \mathbf{i}_{\alpha_{p}}+n_{\alpha_{p}} I_{p}^{m 1} \mathbf{i}_{\beta_{p}}+I_{p}^{m 2} \mathbf{i}_{\varphi}, \quad p=1,2 .
$$

В формулах (35) и (36) $h_{\Sigma_{p}}=h_{\alpha_{p}}=h_{\beta_{p}}$ и $h_{\varphi}$ - коэффициенты Ламе и $\mathbf{i}_{\alpha_{p}}, \mathbf{i}_{\beta_{p}}, \mathbf{i}_{\varphi}-$ единичные орты выбранной системы координат, $n_{\alpha_{p}}, n_{\beta_{p}}-$ координаты нормалей к поверхности $\Sigma_{p}$. В формуле (35) через $\dot{\alpha}_{\Sigma_{p}}$ и $\dot{\beta}_{\Sigma_{p}}$ обозначены производные по параметру $\beta^{\prime}$.

Далее подставим формулы (32)-(34) в систему уравнений (30) и (31) и спроецируем полученные равенства на базис Фурье по угловой координате $\varphi$. Принимая во внимание соотношения (35) и (36), получим следующую бесконечную систему одномерных интегральных уравнений:

$$
\mathbf{K}_{11}^{m} \mathbf{I}_{1}^{m}+\mathbf{K}_{12}^{n} \mathbf{I}_{2}^{m}=\mathbf{B}_{1}^{m},
$$

$$
\mathbf{K}_{21}^{m} \mathbf{I}_{1}^{m}+\mathbf{K}_{22}^{m} \mathbf{I}_{2}^{m}=\mathbf{B}_{2}^{m}, \quad m=0, \pm 1, \pm 2, \ldots,
$$

где $\mathbf{I}_{p}^{m}=\left[I_{p}^{m 1}, I_{p}^{m 2}\right]^{T}, \mathbf{B}_{p}^{m}=\left[B_{p}^{m 1}, B_{p}^{m 2}\right]^{T}, p=1,2$. В системе (37) вводятся следующие матричные операторы:

$$
\begin{gathered}
\mathbf{K}_{p q}^{m} \mathbf{I}_{q}^{m}=\left(\begin{array}{ll}
\int_{0}^{\pi} K_{p q, 11}^{m}\left(\beta, \beta^{\prime}\right) I_{q}^{m 1}\left(\beta^{\prime}\right) d \beta^{\prime} & \int_{0}^{\pi} K_{p q, 12}^{m}\left(\beta, \beta^{\prime}\right) I_{q}^{m 2}\left(\beta^{\prime}\right) d \beta^{\prime} \\
\int_{0}^{\pi} K_{p q, 21}^{m}\left(\beta, \beta^{\prime}\right) I_{q}^{m 1}\left(\beta^{\prime}\right) d \beta^{\prime} & \int_{0}^{\pi} K_{p q, 22}^{m}\left(\beta, \beta^{\prime}\right) I_{q}^{m 2}\left(\beta^{\prime}\right) d \beta^{\prime}
\end{array}\right), \\
p=1,2 .
\end{gathered}
$$

Ядра в (38) имеют вид

$$
\begin{gathered}
K_{11,11}^{m}=n_{\alpha}\left(n_{\alpha_{1}} e_{1,22}^{m}-n_{\beta_{1}} e_{1,21}^{m}\right)-n_{\beta}\left(n_{\alpha_{1}} e_{1,12}^{m}-n_{\beta_{1}} e_{1,11}^{m}\right), \\
K_{11,12}^{m}=n_{\alpha} e_{1,23}^{m}-n_{\beta} e_{1,13}^{m}, \\
K_{11,21}^{m}=n_{\alpha_{1}} e_{1,32}^{m}-n_{\beta_{1}} e_{1,31}^{m}, \quad K_{11,22}^{m}=e_{1,33}^{m}, \\
K_{12,11}^{m}=-\left(n_{\alpha}\left(n_{\alpha_{2}} e_{2,22}^{m}-n_{\beta_{2}} e_{2,21}^{m}\right)-n_{\beta}\left(n_{\alpha_{2}} e_{2,12}^{m}-n_{\beta_{2}} e_{2,11}^{m}\right)\right), \\
K_{12,12}^{m}=-\left(n_{\alpha} e_{2,23}^{m}-n_{\beta} e_{2,13}^{m}\right), \\
K_{12,21}^{m}=-\left(n_{\alpha_{2}} e_{2,32}^{m}-n_{\beta_{2}} e_{2,31}^{m}\right), \quad K_{12,22}^{m}=-e_{2,33}^{m},
\end{gathered}
$$

где $n_{\alpha}, n_{\beta}$ - координаты нормали к поверхности $S$. Формулы для остальных ядер в системе интегральных уравнений (37) получаются заменой $e_{p, j l}^{m}$ на $h_{p, j l}^{m}$ в равенствах (39), (40). Величины $e_{p, j l}^{m}$ и $h_{p, j l}^{m}$ представляют собой элементы матричных функций $\mathbf{G}_{p, m}^{E}$ или $\mathbf{G}_{p, m}^{H}$, заданных в соответствующих ортогональных координатах. Эти величины наиболее просто могут быть выражены в цилиндрических координатах. При этом удобно представить $\mathbf{G}_{1, m}^{E}$ и $\mathbf{G}_{1, m}^{H}$ в виде суммы:

$$
\begin{gathered}
\mathbf{G}_{1, m}^{E}=\mathbf{G}_{1, m}^{E, s}+\mathbf{G}_{1, m}^{E, r}, \\
\mathbf{G}_{1, m}^{H}=\mathbf{G}_{1, m}^{H, s}+\mathbf{G}_{1, m}^{H, r},
\end{gathered}
$$

где $\mathbf{G}_{1, m}^{E, s}, \mathbf{G}_{1, m}^{H, s}-$ части $Ф Г$, соответствующие полю точечного источника в однородной среде с волновым 


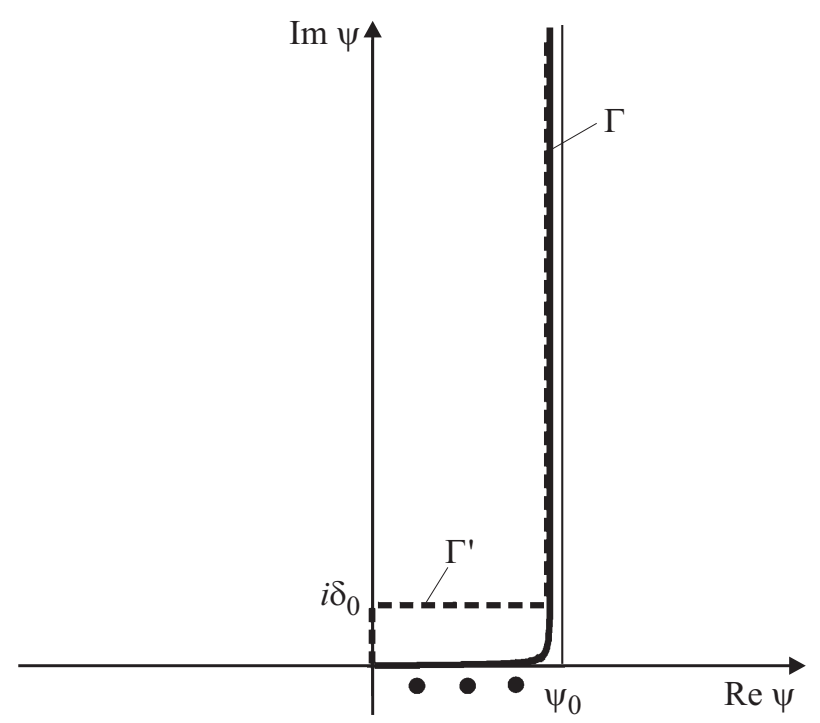

Рис. 2. Комплексная плоскость $\psi$.

числом $k_{2}$ (сингулярные части ФГ, определяемые аналогично формулам (9)-(11)) и $\mathbf{G}_{1, m}^{E, r}, \mathbf{G}_{1, m}^{H, r}-$ добавочные части ФГ, обусловленные наличием границы раздела сред. Подробные выражения для элементов $Ф Г \mathbf{G}_{1, m}^{E, s}$, $\mathbf{G}_{1, m}^{H, s}$ а также функций $\mathbf{G}_{2, m}^{E}, \mathbf{G}_{2, m}^{H}$ приведены в работе [11]. Элементы добавочных $\Phi \Gamma \mathbf{G}_{1, m}^{E, r}, \mathbf{G}_{1, m}^{H, r}$ получаются из формул (12)-(29).

Сделаем замечание относительно вычисления интегралов Зоммерфельда, через которые выражаются добавочные части $Ф \Gamma \mathbf{G}_{1, m}^{E, r}, \mathbf{G}_{1, m}^{H, r}$. Если среда внутри диэлектрического слоя имеет небольшие потери или вообще не имеет потерь, возникает проблема при вычислении интегралов Зоммерфельда из-за того, что особенности (полюсы) подынтегральных функций в (21)-(23) расположены около действительной оси переменной $\kappa$ (или на самой оси). Чтобы преодолеть эту трудность, мы осуществляли замену переменной по формуле $\kappa=k_{2} \sin \psi$ в указанных интегралах. В результате переходили к интегрированию по контуру Г, показанному на рис. 2 сплошной линией. На рисунке значение $\psi_{0}=-\operatorname{arctg}\left(k_{2}^{\prime} / k_{2}^{\prime \prime}\right)$, где $k_{2}=k_{2}^{\prime}+i k_{2}^{\prime \prime}$. Точками на рис. 2 показаны полюсы подынтегральных функций. Далее удобно перейти к интегрированию по контуру $\Gamma^{\prime}$, который изображен штриховой линией на рис. 2. В этом случае параметр $\delta_{0}$ имеет порядок $10^{-1}$. Как показывают расчеты, при таком подходе не возникает сложностей при нахождении интегралов Зоммерфельда.

В качестве выходной характеристики задачи можно рассмотреть диаграмму рассеяния $\mathbf{F}(\theta, \varphi)$ волнового поля. Диаграмма рассеяния определяется по формуле

$$
\mathbf{E}^{1} \approx \mathbf{F}(\theta, \varphi) \frac{\exp \left(-i k_{1} r\right)}{r}
$$

при $r \rightarrow \infty$ (здесь $(r, \theta, \varphi)-$ сферические координаты). Для нахождения диаграммы перейдем к асимптотике рассеянного поля в верхнем и нижнем полупространствах. Применяя метод перевала к элементам матричной ФГ, нетрудно показать, что диаграмма рассеяния имеет вид

$$
\mathbf{F}(\theta, \varphi)=\sum_{m=-\infty}^{\infty} \mathbf{f}_{m}(\theta) \exp (i m \varphi)
$$

где $[11,12]$

$$
\mathbf{f}_{m}(\theta)=\int_{0}^{\pi} \mathbf{F}_{m}\left(\theta, \theta^{\prime}\left(\beta^{\prime}\right)\right) \mathbf{T}_{2}^{T}\left(\theta^{\prime}\left(\beta^{\prime}\right)\right) \mathbf{T}_{1}\left(\beta^{\prime}\right) \mathbf{I}_{p}^{m}\left(\beta^{\prime}\right) d \beta^{\prime} .
$$

Здесь $\mathbf{T}_{2}-$ это матрица

$$
\mathbf{T}_{2}=\left(\begin{array}{ccc}
\sin \theta^{\prime} & \cos \theta^{\prime} & 0 \\
0 & 0 & 1 \\
\cos \theta^{\prime} & -\sin \theta^{\prime} & 0
\end{array}\right),
$$

а элементы матрицы $\mathbf{T}_{1}$ приведены в работах $[11,12]$. Матрица $\mathbf{F}_{m}\left(\theta, \theta^{\prime}\right)$ имеет следующие элементы:

$$
\begin{aligned}
f_{m}^{21}= & \pm i^{m} k_{1} \cos \theta\left(\sin \theta^{\prime}\left( \pm i C_{11} \cos \theta-C_{31} k_{1} \sin ^{2} \theta\right)\right. \\
\times & \left.\frac{J_{m+1}\left(\kappa \rho^{\prime}\right)-J_{m-1}\left(\kappa \rho^{\prime}\right)}{2}-C_{33} \cos \theta^{\prime} \sin \theta J_{m}\left(\kappa \rho^{\prime}\right)\right) \\
\times & \exp \left( \pm i k_{1} z^{\prime} \cos \theta\right) \\
f_{m}^{22}= & \pm i^{m} k_{1} \cos \theta\left(\cos \theta^{\prime}\left( \pm i C_{11} \cos \theta-C_{31} k_{1} \sin ^{2} \theta\right)\right. \\
\times & \left.\frac{J_{m+1}\left(\kappa \rho^{\prime}\right)-J_{m-1}\left(\kappa \rho^{\prime}\right)}{2}+C_{33} \sin \theta^{\prime} \sin \theta J_{m}\left(\kappa \rho^{\prime}\right)\right) \\
\times & \exp \left( \pm i k_{1} z^{\prime} \cos \theta\right), \\
f_{m}^{23}= & \mp i^{m} k_{1} \cos \theta\left( \pm C_{11} \cos \theta+i C_{31} k_{1} \sin { }^{2} \theta\right) \\
& \times \frac{J_{m+1}\left(\kappa \rho^{\prime}\right)+J_{m-1}\left(\kappa \rho^{\prime}\right)}{2} \exp \left( \pm i k_{1} z^{\prime} \cos \theta\right) \\
f_{m}^{33}= & \pm i^{m+1} k_{1} C_{11} \cos \theta \\
f_{m}^{31}= & \pm i^{m} k_{1} C_{11} \cos \theta \sin \theta^{\prime} \\
& \times \frac{J_{m+1}\left(\kappa \rho^{\prime}\right)+J_{m-1}\left(\kappa \rho^{\prime}\right)}{2} \exp \left( \pm i k_{1} z^{\prime} \cos \theta\right) \\
f_{m}^{32}= & \pm i k_{1} C_{11} \cos \theta \cos \theta^{\prime} \\
& \times \frac{J_{m+1}\left(\kappa \rho^{\prime}\right)+J_{m-1}\left(\kappa \rho^{\prime}\right)}{2} \exp \left( \pm i k_{1} z^{\prime} \cos \theta\right)
\end{aligned}
$$

где $\kappa=k_{1} \sin \theta$. Остальные элементы равны нулю. В формулах (46)-(51) верхние знаки соответствуют диаграм- 
ме рассеяния в верхнем полупространстве. Далее

$$
\begin{aligned}
& C_{11}=-\frac{i \exp \left(i \gamma_{1} d_{2}\right)}{2 \gamma_{2}}\left(\exp \left(-i \gamma_{2}\left(d_{2}-z^{\prime}\right)\right)\left(1+\frac{R_{\mu}}{\Delta_{\mu}}\right)\right. \\
& +\left(\exp \left(-i \gamma_{2}\left(2 h-d_{2}-z^{\prime}\right)\right)+R_{\mu} \exp \left(-i \gamma_{2}\left(2 h+d_{2}-z^{\prime}\right)\right)\right. \\
& \left.\left.+R_{\mu} \exp \left(-i \gamma_{2}\left(2 h-d_{2}+z^{\prime}\right)\right)\right) \frac{R_{\mu}}{\Delta_{\mu}}\right)
\end{aligned}
$$$$
C_{31}=\mu_{12} \exp \left(i \gamma_{1} d_{2}\right)\left(A_{31} \exp \left(-i \gamma_{2} d_{2}\right)+B_{31} \exp \left(i \gamma_{2} d_{2}\right)\right)
$$

в случае диаграммы в верхнем полупространстве и

$$
\begin{aligned}
& C_{11}=-\frac{i \exp \left(i \gamma_{1} d_{1}\right)}{2 \gamma_{2}}\left(\exp \left(-i \gamma_{2}\left(d_{1}+z^{\prime}\right)\right)\left(1+\frac{R_{\mu}}{\Delta_{\mu}}\right)\right. \\
& +\left(\exp \left(-i \gamma_{2}\left(2 h-d_{1}-z^{\prime}\right)\right)+R_{\mu} \exp \left(-i \gamma_{2}\left(2 h-d_{1}-z^{\prime}\right)\right)\right. \\
& \left.\left.+R_{\mu} \exp \left(-i \gamma_{2}\left(2 h+d_{1}+z^{\prime}\right)\right)\right) \frac{R_{\mu}}{\Delta_{\mu}}\right),
\end{aligned}
$$$$
C_{31}=\mu_{12} \exp \left(i \gamma_{1} d_{1}\right)\left(A_{31} \exp \left(i \gamma_{2} d_{1}\right)+B_{31} \exp \left(-i \gamma_{2} d_{1}\right)\right)
$$

в случае диаграммы в нижнем. В формулах (52)-(55)

$$
\begin{aligned}
& \gamma_{1}= k_{1} \cos \theta, \quad \gamma_{2}=\sqrt{k_{2}^{2}-k_{1}^{2} \sin ^{2} \theta}, \quad R_{\mu}=\frac{\mu_{12} \gamma_{2}-\gamma_{1}}{\mu_{12} \gamma_{2}+\gamma_{1}} \\
& \Delta_{\mu}=1-R_{\mu}^{2} \exp \left(-i 2 \gamma_{2} h\right), \quad h=d_{1}+d_{2}, \\
& A_{31}=-\frac{q_{12}\left(1+R_{\mu}\right) \exp \left(-i \gamma_{2} d_{1}\right)}{2 \gamma_{2} \mu_{12}\left(\gamma_{1}+\varepsilon_{12} \gamma_{2}\right) \Delta_{\mu} \Delta_{\varepsilon}}\left(\exp \left(-i \gamma_{2}\left(d_{1}+z^{\prime}\right)\right)\right. \\
&+R_{\mu} \exp \left(-i \gamma_{2}\left(2 h-d_{1}-z^{\prime}\right)\right) \\
&-R_{\varepsilon} \exp \left(-i \gamma_{2} h\right)\left(\exp \left(-i \gamma_{2}\left(d_{2}-z^{\prime}\right)\right)\right. \\
&\left.\left.+R_{\mu} \exp \left(-i \gamma_{2}\left(2 h-d_{2}+z^{\prime}\right)\right)\right)\right), \\
& B_{31}=-\frac{q_{12}\left(1+R_{\mu}\right) \exp \left(-i \gamma_{2} d_{1}\right)}{2 \gamma_{2} \mu_{12}\left(\gamma_{1}+\varepsilon_{12} \gamma_{2}\right) \Delta_{\mu} \Delta_{\varepsilon}}\left(\exp \left(-i \gamma_{2}\left(d_{2}-z^{\prime}\right)\right)\right. \\
&+R_{\mu} \exp \left(-i \gamma_{2}\left(2 h-d_{2}+z^{\prime}\right)\right) \\
&-R_{\varepsilon} \exp \left(-i \gamma_{2} h\right)\left(\exp \left(-i \gamma_{2}\left(d_{1}+z^{\prime}\right)\right)\right. \\
&\left.\left.+R_{\mu} \exp \left(-i \gamma_{2}\left(2 h-d_{1}-z^{\prime}\right)\right)\right)\right), \\
& \mu_{12}=\frac{\mu_{1}}{\mu_{2}}, \quad \varepsilon_{12}=\frac{\varepsilon_{1}}{\varepsilon_{2}}, \quad q_{12}=\varepsilon_{12} \mu_{12}-1
\end{aligned}
$$

Выражения для величин $R_{\varepsilon}, \Delta_{\varepsilon}$ получаются заменой $\mu_{12}$ на $\varepsilon_{12}$ в формулах для $R_{\mu}, \Delta_{\mu}$, а представления для коэффициентов $C_{33}$ получаются заменой $R_{\mu}, \Delta_{\mu}$ на $R_{\varepsilon}$, $\Delta_{\varepsilon}$ в формулах (52) и (54).

\section{3. Решение задачи дифракции при помощи ММОИу}

Рассмотрим задачу дифракции поля плоской волны вида (3) или (4) на теле вращения, расположенном в однородном диэлектрическом слое. Геометрия задачи вновь иллюстрируется рис. 1. Как и выше, будем считать, что диэлектрические проницаемости среды слоя и окружающего пространства равны $\varepsilon_{2}$ и $\varepsilon_{1}$, а $\varepsilon_{i}=\varepsilon_{i}(\rho, z)-$ диэлектрическая проницаемость среды внутри тела, которая не зависит от угла $\varphi$ (система координат та же, что и в первом разделе). Таким образом, в рассматриваемом случае рассеиватель имеет переменные материальные характеристики. В отличие от задачи, исследованной в предыдущем разделе, будем предполагать среду во всем пространстве магнитооднородной, причем всюду магнитная проницаемость $\mu=1$. Граничные условия на поверхности тела $S$, на границах раздела и условия излучения остаются прежними.

Используя векторный аналог формулы Грина [14], нетрудно показать, что вектор напряженности рассеянного электрического поля магнитооднородной слоистой среды представляется в виде

$$
\mathbf{E}^{1}(\mathbf{r})=\int_{D}\left(k_{i}^{2}\left(\mathbf{r}^{\prime}\right)-k_{2}^{2}\right) \mathbf{G}_{1}^{E}\left(\mathbf{r}, \mathbf{r}^{\prime}\right) \mathbf{E}\left(\mathbf{r}^{\prime}\right) d V^{\prime} .
$$

Поместим далее точку наблюдения в область $D$, в результате чего получим, что полное электрическое поле удовлетворяет следующему интегральному уравнению 2-го рода [14]:

$$
\mathbf{E}(\mathbf{r})+\int_{d}\left(k_{2}^{2}-k_{i}^{2}\left(\mathbf{r}^{\prime}\right)\right) \mathbf{G}_{1}^{E}\left(\mathbf{r}, \mathbf{r}^{\prime}\right) \mathbf{E}\left(\mathbf{r}^{\prime}\right) d V^{\prime}=\mathbf{E}^{0}(\mathbf{r}), \quad \mathbf{r} \in D,
$$

где $k_{i}=\omega \sqrt{\varepsilon_{i}\left(\mathbf{r}^{\prime}\right)}$ - волновое число среды внутри тела. При этом ФГ $\mathbf{G}_{1}^{E}$ разбивается на сумму сингулярной $\mathbf{G}_{1}^{E, s}$ и регулярной $\mathbf{G}_{1}^{E, r}$ частей, причем функция $\mathbf{G}_{1}^{E, s}$ выражается через скалярную ФГ $G\left(\mathbf{r}, \mathbf{r}^{\prime}\right)=\frac{\exp \left(-i k_{2} R\right)}{4 \pi R}$, т. е. определяется по формулам, аналогичным (9)-(11). В соответствии с ММОИУ [9] заменим ФГ свободного пространства $G\left(\mathbf{r}, \mathbf{r}^{\prime}\right)$ на функцию $G_{\delta}\left(\mathbf{r}, \mathbf{r}^{\prime}\right)=\frac{\exp \left(-i k_{2} R_{\delta}\right)}{4 \pi R_{\delta}}$, где $R_{\delta}=\sqrt{\rho^{2}+\rho^{\prime 2}-2 \rho \rho^{\prime} \cos \psi+\left(z-z^{\prime}\right)^{2}+\delta^{2}}, \quad \delta>0$ малый параметр. Таким образом, ядро уравнения (61) не будет иметь особенностей при совпадении аргументов. Как указано во Введении, данный подход аналогичен МПГУ [10], который состоит в замене сингулярного ядра интегрального уравнения на близкое ядро. При этом вместо сингулярного интегрального уравнения получаем уравнение с ядром, не имеющим особенности.

Как и в предыдущем разделе, для алгебраизации задачи разложим неизвестную функцию, первичное поле и ФГ в ряды Фурье и подставим эти разложения в уравнение (61). В результате получим бесконечный 
набор двумерных интегральных уравнений для гармоник Фурье вектора напряженности электрического поля:

$$
\begin{aligned}
& \mathbf{E}_{m}(\rho)+\int_{\tilde{D}}\left(k_{2}^{2}-k_{i}^{2}\left(\boldsymbol{\rho}^{\prime}\right)\right) \mathbf{G}_{1, m}^{E}\left(\boldsymbol{\rho}, \boldsymbol{\rho}^{\prime}\right) \mathbf{E}_{m}\left(\boldsymbol{\rho}^{\prime}\right) \rho^{\prime} d \rho^{\prime} d z= \\
& \quad=\mathbf{E}_{m}^{0}(\rho), \quad \boldsymbol{\rho} \in \tilde{D} .
\end{aligned}
$$

Здесь $m=0, \pm 1, \pm 2 \ldots, \boldsymbol{\rho}=(\rho, z), \tilde{D}-$ двумерная область, получаемая в осевом сечении области $D$ тела, $\mathbf{E}_{m}(\boldsymbol{\rho})$ - гармоники электрического поля. Для численного решения уравнений (62) будем предполагать, что область $\tilde{D}$ задана уравнениями

$$
\rho=\rho(u, v), \quad z=z(u, v),
$$

где $u_{1} \leq u \leq u_{2}$. Таким образом, имеется отображение области $D$ на прямоугольник: $\tilde{D}^{\prime}=\left[u_{1}, u_{2}\right] \times\left[v_{1}, v_{2}\right]$. В новых переменных интегральные уравнения (62) могут быть записаны в виде

$$
\begin{aligned}
& \mathbf{E}_{m}(u, v)+\int_{u_{1}}^{u_{2}} \int_{v_{1}}^{v_{2}}\left(k_{2}^{2}-k_{i}^{2}\left(u^{\prime}, v^{\prime}\right)\right) \mathbf{G}_{1, m}^{E}\left(u, v, u^{\prime}, v^{\prime}\right) \\
& \quad \times \mathbf{E}_{m}\left(u^{\prime}, v^{\prime}\right)\left|J\left(u^{\prime}, v^{\prime}\right)\right| \rho^{\prime}\left(u^{\prime}, v^{\prime}\right) d u^{\prime} d v^{\prime}=\mathbf{E}_{m}^{0}(u, v),
\end{aligned}
$$

где $m=0, \pm 1, \pm 2, \ldots, J\left(u^{\prime}, v^{\prime}\right)$ - якобиан преобразования (63). Для решения уравнений (64) использовали метод Крылова-Боголюбова аналогично работе [9]. В результате применения данного метода система уравнений (64) сводится к бесконечному набору линейных алгебраических систем относительно неизвестных коэффициентов $\mathbf{c}_{j l}^{m}$ разложения величин $\mathbf{E}_{m}(u, v)$ по базису, состоящему из кусочно-постоянных функций [9]:

$$
\begin{gathered}
\sum_{j=1}^{N_{1}} \sum_{l=1}^{N_{2}} \mathbf{G}_{s t}^{j l, m} \mathbf{c}_{j l}^{m}=\mathbf{w}_{s t}^{m}, \\
s=1,2, \ldots, N_{1}, \quad t=1,2, \ldots, N_{2} .
\end{gathered}
$$

Здесь

$$
\begin{gathered}
\mathbf{G}_{s t}^{j l, m}=\delta_{s j} \delta_{t l}+\int_{\bar{u}_{j}-\Delta_{1} \bar{v}_{l}-\Delta_{2}}^{\bar{u}_{j}+\Delta_{1} \bar{v}_{l}+\Delta_{2}}\left(k_{2}^{2}-k_{i}^{2}(u, v)\right) \\
\times \mathbf{G}_{1, m}^{E}\left(\bar{u}_{s}, \bar{v}_{t}, u, v\right)|J(u, v)| \rho(u, v) d u d v, \\
\mathbf{w}_{s t}^{m}=\mathbf{E}_{m}^{0}\left(\bar{u}_{s}, \bar{v}_{t}\right),
\end{gathered}
$$

$\delta_{m n}$ - символ Кронекера. В системах (65) точки коллокации определяются из соотношений

$$
\bar{u}_{s}=u_{1}+(2 s-1) \Delta_{1}, \quad s=1,2, \ldots, N_{1},
$$

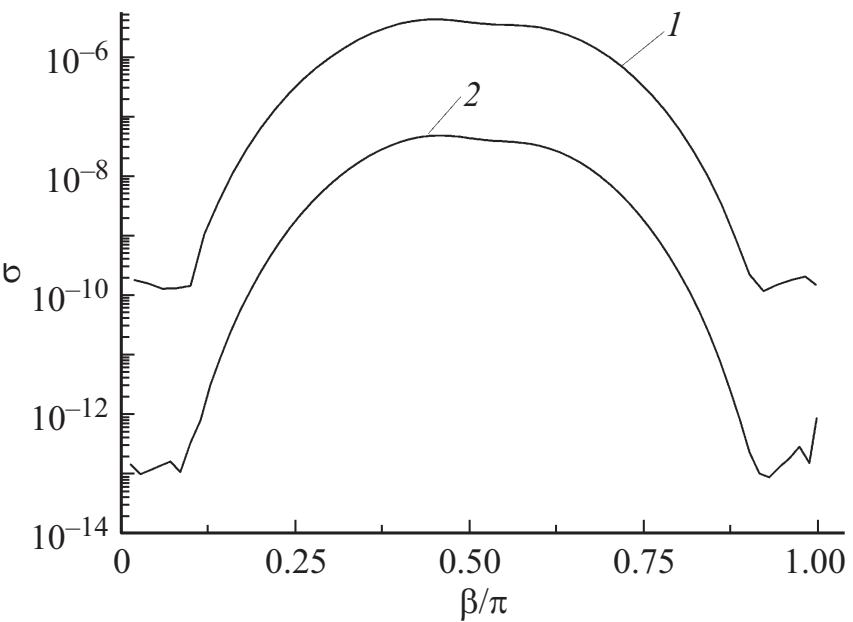

Рис. 3. Распределение невязки на контуре осевого сечения сфероида. Кривые 1 и 2 соответствуют числу дискретных источников и гармоник $N=50, M=9$ и $N=70, M=11$.

$$
\bar{v}_{t}=v_{1}+(2 t-1) \Delta_{2}, \quad t=1,2, \ldots, N_{2},
$$

где $\Delta_{1}=\frac{u_{2}-u_{1}}{2 N_{1}}, \Delta_{2}=\frac{v_{2}-v_{1}}{2 N_{2}}$.

Матричные элементы (66) вычислялись при помощи следующего алгоритма. Воспользуемся тем, что ФГ $\mathbf{G}_{1, m}^{E}$ имеет вид (41). При этом величины $\mathbf{G}_{1, m}^{E, r}$ являются медленно меняющимися функциями координат, а сингулярная часть $\mathbf{G}_{1, m}^{E, s}$ меняется очень быстро при приближении точки наблюдения к точке источника. Тогда можно находить интегралы в (66) по приближенной формуле

$$
\begin{aligned}
& \mathbf{G}_{s t}^{j l, m}=\delta_{s j} \delta_{t l}+\int_{\bar{u}_{j}-\Delta_{1}}^{\bar{u}_{j}+\Delta_{1}-\Delta_{2}} \int_{\bar{v}_{l}+\Delta_{2}}\left(k_{2}^{2}-k_{i}^{2}(u, v)\right) \mathbf{G}_{1, m}^{E, s}\left(\bar{u}_{s}, \bar{v}_{t}, u, v\right) \\
& \times|J(u, v)| \rho(u, v) d u d v+4\left(k_{2}^{2}-k_{i}^{2}\left(\bar{u}_{j}, \bar{v}_{l}\right)\right) \\
& \quad \times \mathbf{G}_{1, m}^{E, r}\left(\bar{u}_{s}, \bar{v}_{t}, \bar{u}_{j}, \bar{v}_{l}\right)\left|J\left(\bar{u}_{j}, \bar{v}_{l}\right)\right| \rho\left(\bar{u}_{j}, \bar{v}_{l}\right) \Delta_{1} \Delta_{2} .
\end{aligned}
$$

Как следует из формулы (70) и формул для ФГ предыдущего раздела, добавочные матричные элементы, обусловленные регулярной частью ФГ, выражаются в виде однократных интегралов (интегралов Зоммерфельда). Кроме того, аналогично работе [9] повторные интегралы в (70) следует вычислять более точно только для диагональных элементов матрицы. Интегралы во внедиагональных элементах можно находить, например, применяя формулы Гаусса с небольшим числом узлов. При таком подходе удается существенно сократить время вычисления матричных элементов на ЭВМ. 
При использовании ММОИУ диаграмма рассеяния вновь имеет вид (43), причем

$$
\begin{aligned}
\mathbf{F}_{m}(\theta)= & \sum_{j=1}^{N_{1}} \sum_{l=1}^{N_{2}}\left(\int_{\bar{u}_{j}-\Delta_{1}}^{\bar{u}_{j}+\Delta_{1}} \int_{\bar{v}_{l}-\Delta_{2}}^{\bar{v}_{l}+\Delta_{2}}\left(k_{i}^{2}(u, v)-k_{2}^{2}\right) \mathbf{F}_{m}\left(\theta, \theta^{\prime}(u, v)\right)\right. \\
& \left.\times \mathbf{T}_{2}^{T}\left(\theta^{\prime}(u, v)\right)|J(u, v)| \rho^{\prime}(u, v) d u d v\right) \mathbf{c}_{j l}^{m}
\end{aligned}
$$

Отметим, что для численной оценки интегралов в формуле (71) можно вновь использовать двумерный аналог формулы прямоугольников. Таким образом, вычисление диаграммы (после определения токов на вспомогательных поверхностях $\Sigma_{1}$ и $\Sigma_{2}$ или поля внутри тела) не требует большого объема вычислений в случае использования как ММДИ, так и ММОИУ.

\section{4. Результаты расчетов}

Рассмотрим результаты численного моделирования. На рис. 3 и 4 приведены зависимости невязки граничных условий (1) на контуре осевого сечения однородного сфероида и однородного суперэллипсоида вращения, расположенного в диэлектрическом слое. Уравнение контура осевого сечения суперэллипсоида имеет вид

$$
\left(\frac{\rho}{a}\right)^{2 q}+\left(\frac{z}{b}\right)^{2 q}=1
$$

Решение задачи осуществлялось с использованием метода на основе ММДИ (первый метод). Невязка определяется по формулам

$$
\sigma=\sqrt{\sigma_{1}^{2}+\sigma_{2}^{2}}
$$

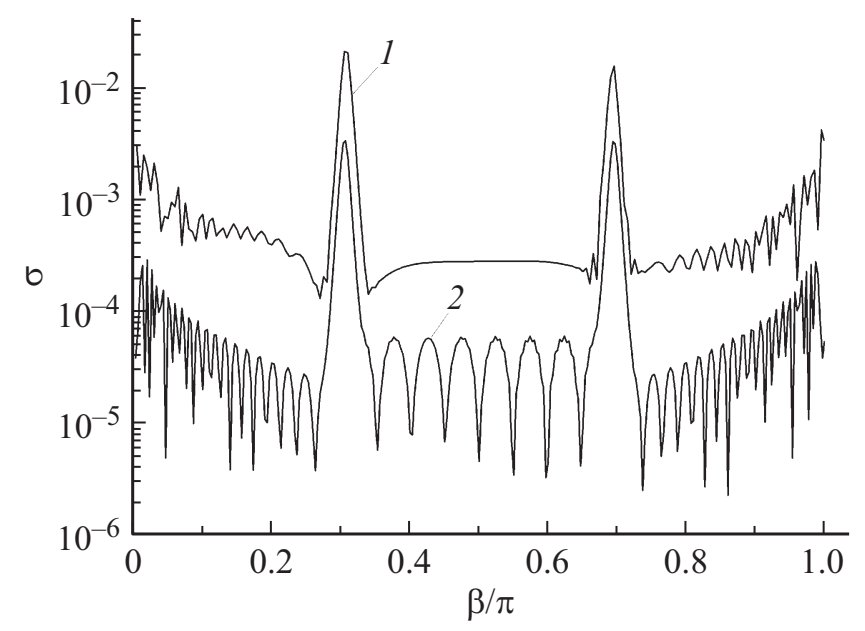

Рис. 4. Распределение невязки на контуре осевого сечения суперэллипсоида. Кривые 1 и 2 соответствуют числу дискретных источников и гармоник $N=200, M=9$ и $N=300, M=11$.
Сравнение результатов расчета диаграммы рассеяния, полученных при помощи ММДИ И ММОИУ

\begin{tabular}{c|c|c|c|c}
\hline \multirow{2}{*}{$\begin{array}{c}\text { Уол } \theta, \\
\text { deg }\end{array}$} & \multicolumn{2}{|c|}{ Дифракция на сфероиде } & \multicolumn{2}{|c}{ Дифракция на цилиндре } \\
\cline { 2 - 5 } & $\begin{array}{c}\text { Метод } \\
\text { наснове } \\
\text { ММДИ }\end{array}$ & $\begin{array}{c}\text { Метод } \\
\text { на основе } \\
\text { ММОИУ }\end{array}$ & $\begin{array}{c}\text { Метод } \\
\text { на основе } \\
\text { ММДИ }\end{array}$ & $\begin{array}{c}\text { Метод } \\
\text { на основе } \\
\text { ММОИУ }\end{array}$ \\
\hline 0 & 2.4629 & 2.4651 & 2.7530 & 2.7570 \\
36 & 4.0816 & 4.0709 & 5.8789 & 5.8990 \\
72 & 1.5084 & 1.5039 & 2.2079 & 2.2125 \\
108 & 1.0271 & 1.0235 & 1.3749 & 1.3782 \\
144 & 1.9749 & 1.9714 & 3.0124 & 3.0337 \\
180 & 1.0381 & 1.0458 & 0.8707 & 0.8810 \\
216 & 0.2898 & 0.2913 & 0.5379 & 0.5418 \\
252 & 0.1351 & 0.1362 & 0.1413 & 0.1422 \\
288 & 0.2318 & 0.2325 & 0.3086 & 0.3076 \\
324 & 0.3043 & 0.3043 & 0.6767 & 0.6824
\end{tabular}

где

$$
\begin{gathered}
\sigma_{p}(\beta, \varphi)=\| \sum_{m=-M}^{M}\left(\mathbf{K}_{p 1}^{m} \mathbf{I}_{1}^{m}+\mathbf{K}_{p 2}^{m} \mathbf{I}_{2}^{m}-\mathbf{B}_{p}^{m}\right) \exp (\operatorname{im\varphi } \varphi) \\
p=1,2 .
\end{gathered}
$$

В формуле (74) используется евклидова норма. В случае задачи дифракции как на сфероиде, так и на суперэллипсоиде для построения вспомогательных поверхностей использовались сплюснутые сфероидальные координаты [11]. Система интегральных уравнений (37) решалась методом коллокации [11,12]. Параметры задачи были следующие. Полуоси сфероида $k_{0} a=1.5708, k_{0} b=3.1416$ (полуось $a$ направлена вдоль оси $z$ ), полуоси суперэллипсоида $k_{0} a=1.5708$ и $k_{0} b=3.1416$, параметр $q=15$, диэлектрические постоянные сред $\varepsilon_{1}=1, \varepsilon_{2}=2.25-i 10^{-3}, \varepsilon_{i}=4$, расстояния $k_{0} d_{1}=k_{0} d_{2}=3.1416$. Здесь $k_{0}=2 \pi / \lambda$, где $\lambda-$ длина волны. Магнитная проницаемость везде равна единице. Угол падения плоской волны (3) $\chi_{1}=45^{\circ}$, а угол $\varphi=0$. Как видно из рисунков, максимальный уровень невязки становится значительно меньше с увеличением числа дискретных источников и угловых гармоник. Максимальное значение невязки не превышает $5 \cdot 10^{-8}$ в случае дифракции на сфероиде (при $N=70$, $M=11)$ и не превышает $5 \cdot 10^{-3}$ в случае дифракции на суперэллипсоиде (при $N=300, M=11$ ). Этот факт гарантирует корректность метода, основанного на ММДИ.

В таблице приведены результаты расчета модуля диаграммы рассеяния для задачи дифракции плоской волны (3) на однородном сфероиде и однородном круговом цилиндре конечных размеров, погруженных в диэлектрический слой. Задача решалась при помощи ММДИ и ММОИУ. При решении задачи дифракции на круговом цилиндре с помощью ММДИ поверхность цилиндра аппроксимировалась поверхностью суперэллипсоида (72) 

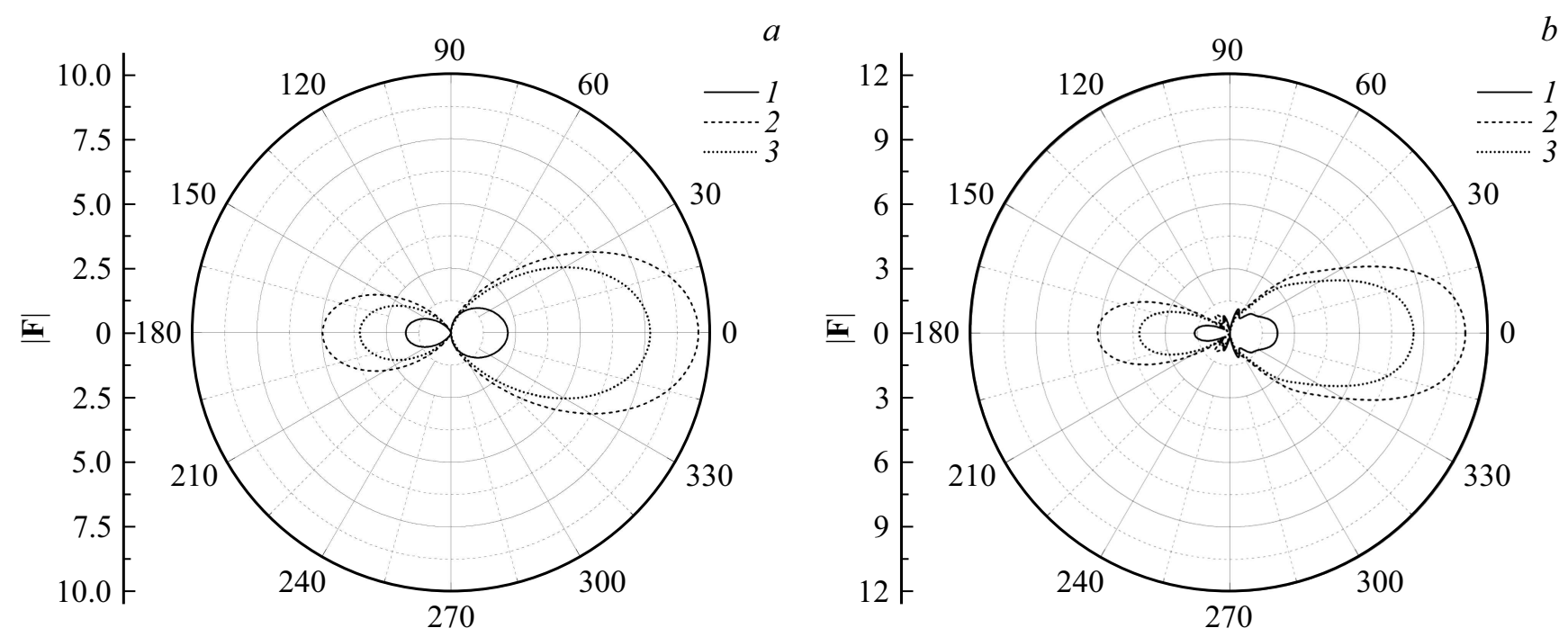

Рис. 5. Угловые зависимости диаграммы для диэлектрического неоднородного цилиндра Расстояния до границ слоя $k_{0} d_{1}=k_{0} d_{2}=3.1416(a)$ и $k_{0} d_{1}=k_{0} d_{2}=9.4248(b)$. Кривые 1 и 2 соответствуют случаю, когда диэлектрическая проницаемость $\varepsilon_{i}$ изменяется согласно (75) или (76), а кривая 3 соответствует рассеянию на однородном цилиндре с диэлектрической проницаемостью $\varepsilon_{i}=4$.
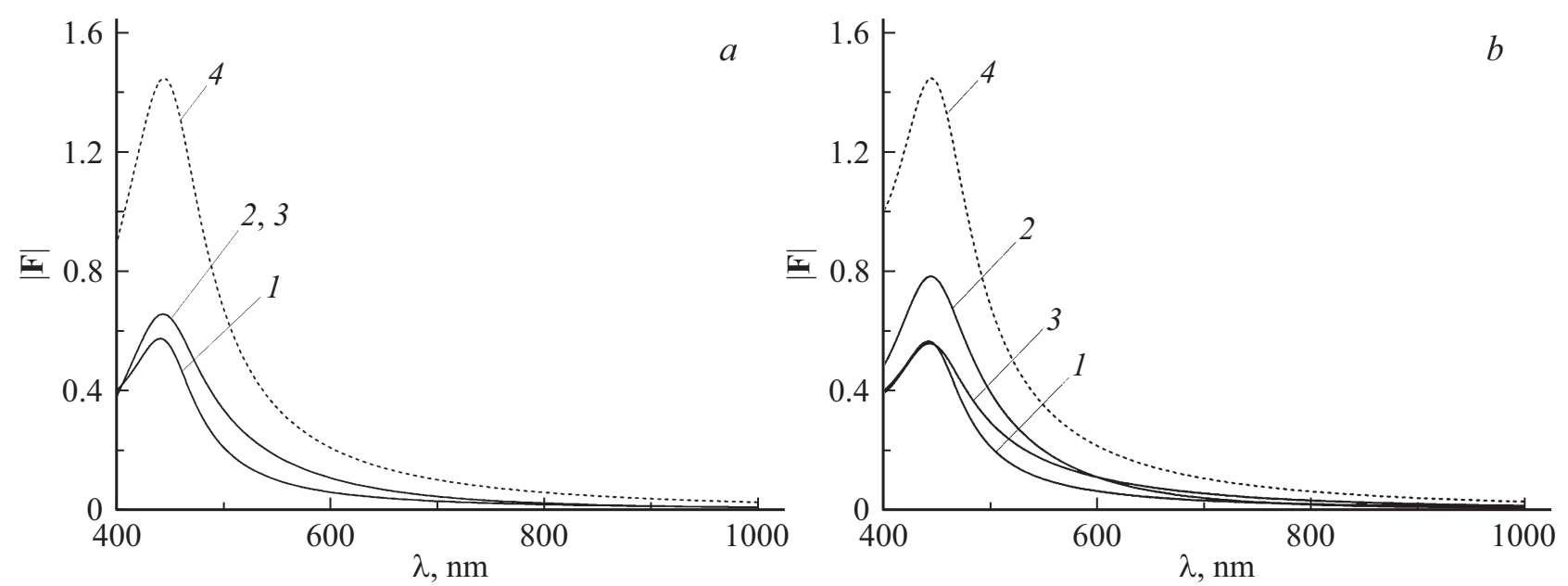

Рис. 6. Зависимости модуля диаграммы рассеяния от длины волны. Дифракция на сфере. Угол наблюдения $\theta=0$ (a) и $\theta=180^{\circ}(b)$. Кривая 1 соответствует случаю $d_{1}=d_{2}=100 \mathrm{~nm}$, кривая 2 соответствует $d_{1}=40$ и $d_{2}=160 \mathrm{~nm}$, кривая 3 соответствует $d_{1}=160 \mathrm{~nm}$ и $d_{2}=40 \mathrm{~nm}$, кривая 4 соответствует дифракции на сфере в однородной среде.

с параметром $q=15$. Размеры тел были следующие: полуоси сфероида $k_{0} a=1.5708, k_{0} b=3.1416$ высота и радиус цилиндра $2 k_{0} a=3.1416, k_{0} b=3.1416$, расстояния $k_{0} d_{1}=k_{0} d_{2}=3.1416$. Диэлектрическая и магнитная проницаемости среды такие же, как указано выше. Угол падения плоской волны (3) $\chi_{1}=45^{\circ}$. Количество дискретных источников $N=50$ в случае дифракции на сфероиде и $N=300$ в случае дифракции на суперэллипсоиде. При решении задачи с помощью ММОИУ использовались обобщенные полярные координаты $(\rho=b u \sin v$, $z=a u \cos v)$ или цилиндрические координаты $(\rho=u$, $z=v)$ в случае дифракции на сфероиде или цилиндре соответственно. Количество точек коллокации $N_{1}=10$,
$N_{2}=25$ в случае дифракции на сфероиде и $N_{1}=15$, $N_{2}=15$ в случае дифракции на цилиндре. Параметр $k_{0} \delta=10^{-3}$. Диаграмма рассчитывалась для углов $\varphi=0$ и $\varphi=180^{\circ}$. Как видно из таблицы, результаты расчета диаграммы, полученные методами на основе ММДИ и ММОИУ, отличаются не более чем на 0.9 и $1.2 \%$ соответственно в случае дифракции на сфероиде и цилиндре. Отметим, что в последнем случае метод, основанный на ММДИ, является приближенным, поскольку мы рассматриваем дифракцию на суперэллипсоиде, а не на цилиндре.

В качестве примера дифракции на неоднородном теле вращения, расположенном в слоистой среде, была рас- 

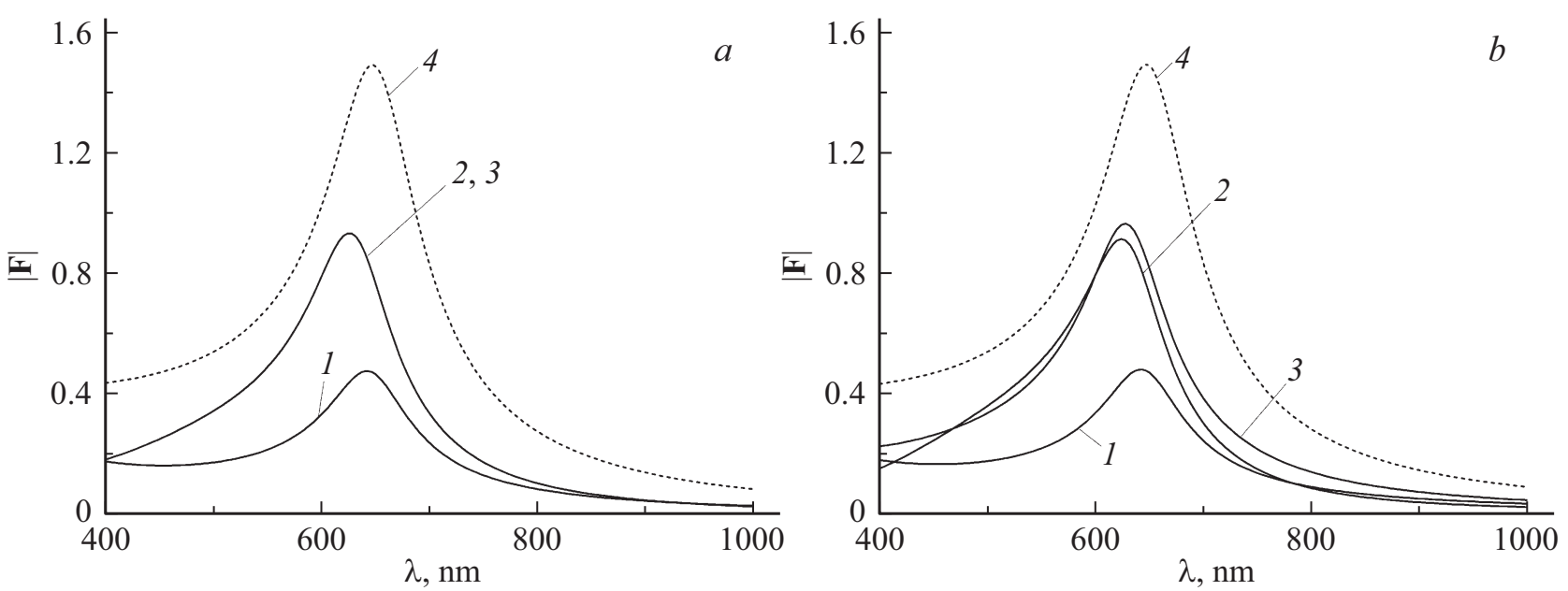

Рис. 7. Зависимости модуля диаграммы рассеяния от длины волны. Дифракция на сфероиде. Угол наблюдения $\theta=0$ ( $a$ ) и $\theta=180^{\circ}(b)$. Кривая 1 соответствует случаю $d_{1}=d_{2}=100 \mathrm{~nm}$, кривая 2 соответствует $d_{1}=21.9055 \mathrm{~nm}$ и $d_{2}=178.0945 \mathrm{~nm}$, кривая 3 соответствует $d_{1}=178.0945 \mathrm{~nm}$ и $d_{2}=21.9055 \mathrm{~nm}$, кривая 4 соответствует дифракции на сфероиде в однородной среде.

смотрена дифракция на неоднородном круговом диэлектрическом цилиндре. Диэлектрическая проницаемость цилиндра изменялась по закону

$$
\varepsilon_{i}(\rho, z)=\frac{\varepsilon_{c}}{\operatorname{ch}^{2}\left(\frac{\rho}{b}\right)}
$$

или

$$
\varepsilon_{i}(\rho, z)=\frac{\varepsilon_{c}}{\operatorname{ch}^{2}\left(\frac{z}{a}\right)},
$$

где $\varepsilon_{c}=5.534$. На рис. 5 приведены угловые зависимости модуля диаграммы рассеяния для случая дифракции плоской волны (3) на рассматриваемом теле, расположенном в диэлектрическом слое. Магнитная проницаемость всюду равнялась единице, а диэлектрические проницаемости окружающего слой пространства и слоя равны $\varepsilon_{1}=1, \varepsilon_{2}=2.25-i 10^{-3}$ соответственно. Размеры цилиндра: высота $2 a=\lambda / 2$ и радиус $b=\lambda / 2$. Рассматривалось нормальное падение плоской волны. Кривые на рисунках построены для полуплоскостей $\varphi=0$ и $\varphi=180^{\circ}$. Отметим, что диэлектрическая проницаемость однородного цилиндра (т.е. $\left.\varepsilon_{i}=4\right)$ равна среднему арифметическому значений проницаемости неоднородного тела, взятых в центре цилиндра и при $\rho=b$ или $z=a$. Решение задачи дифракции осуществлялось с помощью ММОИУ. Из рисунков следует, что в случае дифракции на цилиндре, расположенном в слое с $k_{0} d_{1}=k_{0} d_{2}=3.1416$, диаграмма рассеяния имеет только два лепестка в направлениях $\theta=0$ и $\theta=180^{\circ}$. В случае, когда цилиндр расположен в широком слое (рис. 5,b), диаграмма имеет боковые лепестки. Из рисунков видно, что, несмотря на небольшие размеры цилиндра, диаграммы, полученные для разных зависимостей диэлектрической проницаемости среды внутри тела, различаются существенно. В частности, отличие диаграмм однородного цилиндра и цилиндра, диэлектрическая проницаемость которого изменяется в соответствии с формулой (76), намного меньше, чем различие между диаграммами однородного цилиндра и тела с диэлектрической проницаемостью, которая изменяется в соответствии с формулой (75).

Рассмотрим результаты дифракции на плазмонных частицах $[15,16]$, находящихся в диэлектрическом слое. Представляет интерес изучение резонансных свойств таких структур и сравнение дифракционных характеристик плазмонных частиц, расположенных в слое, с характеристиками рассеивателей, расположенных в однородном пространстве. Следуя $[15,16]$, мы предполагаем, что диэлектрическая проницаемость $\varepsilon_{i}=\varepsilon_{i}^{\prime}+i \varepsilon_{i}^{\prime \prime}$ среды тела зависит от частоты по закону

$$
\begin{aligned}
& \varepsilon_{i}^{\prime}=\varepsilon_{\infty}-\frac{\omega_{p}^{2} \tau^{2}}{1+\omega^{2} \tau^{2}}, \\
& \varepsilon_{i}^{\prime \prime}=-\frac{\omega_{p}^{2} \tau}{\omega\left(1+\omega^{2} \tau^{2}\right)},
\end{aligned}
$$

где $\hbar \omega_{p}=9.1 \mathrm{eV}, \tau=3.6 \cdot 10^{-14} \mathrm{~s}, \varepsilon_{\infty}=3.7, \hbar-$ постоянная Планка. В рассматриваемом случае длина волны и частота связаны соотношением

$$
\lambda(\mathrm{nm})=\frac{1240}{\hbar \omega}
$$

справедливом при $\lambda>400 \mathrm{~nm}$. На рис. 6 и 7 приведены зависимости модуля диаграммы рассеяния в направлениях $\theta=0$ и $\theta=180^{\circ}$ от длины волны. Угол $\varphi=0$. Рисунок 6 относится к дифракции на плазмонной сфере с радиусом $30 \mathrm{~nm}$, а рис. 7 соответствует рассеянию на плазмонном сплюснутом сфероиде с отношением полуосей $a / b=0.25$. Размеры сфероида выбирали так, чтобы объем частицы был равен объему сферы радиусом 
$30 \mathrm{~nm}$. Рассматривалось нормальное падение плоской волны (3). Диэлектрические постоянные среды слоя и окружающего пространства $\varepsilon_{1}=1, \varepsilon_{2}=2.25-i 10^{-3}$. Кривые 4 соответствуют рассеянию на теле, расположенном в однородной среде с диэлектрической проницаемостью $\varepsilon_{1}=2.25$. Магнитная проницаемость всюду равна единице. Из рисунков следует, что зависимости величины $|\mathbf{F}(0,0)|$ от длины волны совпадают, когда частица находится вблизи верхней или нижней границ слоя (кривые 2 и 3 на рисунках). Как видно из рис. 6, в случае дифракции на сфере, расположенной в слое, возникают резонансы на длине волны, приблизительно равной $440 \mathrm{~nm}$. В то же время при дифракции на сфере, расположенной в однородной среде с $\varepsilon_{1}=2.25$, имеется более значительный пик частотной зависимости на той же длине волны. В случае дифракции на сплюснутом сфероиде, расположенном в диэлектрическом слое, возникают резонансы на длине волны, приблизительно равной $630 \mathrm{~nm}$. При дифракции на том же сфероиде, расположенном в свободном пространстве, резонансная длина волны равна примерно $650 \mathrm{~nm}$. Таким образом, максимумы зависимостей диаграммы смещаются в сторону больших длин волн. Из рис. 7 видно, что максимумы зависимостей диаграммы имеют большие значения при дифракции на сфероиде, расположенном в однородном пространстве, чем максимумы соответствующих зависимостей для сфероида, расположенного в слое (как и при дифракции на cфepe).

\section{5. Заключение}

Предложены два подхода решения векторной задачи дифракции на теле вращения, расположенном в однородном диэлектрическом слое. Для проверки методики, основанной на ММДИ, построено распределение невязки граничного условия на контуре осевого сечения однородного сфероида и суперэллипсоида вращения, погруженных в диэлектрический слой. Показано, что максимальный уровень невязки для тела, граница которого имеет участки резкого изменения координат (суперэллипсоид), не превышает $5 \cdot 10^{-3}$. Проведено сравнение обоих методов на примере задачи дифракции на однородном сплюснутом сфероиде и круговом цилиндре конечных размеров, расположенных в диэлектрическом слое. Показано, что результаты расчета диаграммы, полученной методами на основе ММДИ и ММОИУ, отличаются не более чем на 0.9 и $1.2 \%$ соответственно в случае дифракции на сфероиде и цилиндре. Представлены угловые зависимости диаграммы для неоднородного цилиндра, расположенного в диэлектрическом слое. Показано значительное влияние неоднородности среды диэлектрического тела на диаграмму рассеяния. Построены зависимости модуля диаграммы рассеяния от длины волны для плазмонных сферы и сфероида. Продемонстрировано наличие резонансов в таких структурах. Показано, что резонансные пики имеют большее значение в том случае, когда тело находится в однородной среде с той же диэлектрической проницаемостью, что и среда слоя.

\section{Финансирование работы}

Работа выполнена при поддержке Российского фонда фундаментальных исследований (проекты № 18-0200961, 19-02-00654).

\section{Конфликт интересов}

Автор заявляет, что у него нет конфликта интересов.

\section{Список литературы}

[1] Zhuk N.P., Yarovoy A.G. // IEEE Transactions on Antennas and Propagation. 1994. V. AP-42. N 1. P. 16.

[2] Жук Н.П., Шульга С.Н., Яровой А.Г. // ЖТФ. 1998. Т. 68. № 1. C. 84; Zhuk N.P., Shulga S.N., Yarovoy A.G. // Technical Physics. The Russian Journal of Applied Physics. 1998. V. 43. N 1. P. 75.

[3] Панин С.Б., Поединчук А.Е. // Изв. вузов. Радиофизика. 2002. T. 45. № 8. C. 690.

[4] Гришина Н.В., Еремин Ю.А. // Опт. и спектр. 1999. Т. 86. № 3. C. 475; Grishina N.V., Eremin Y.A. // Optics and Spectroscopy. 1999. V. 86. N 3. P. 415.

[5] Гришина Н.В., Еремин Ю.А., Свешников А.Г. // Опт. и спектр. 2014. T. 117. № 6. C. 964; Grishina N.V., Eremin Y.A., Sveshnikov A.G. // Optics and Spectroscopy. 2014. V. 117. N 6. P. 992.

[6] Yurkin M.A., Huntemann M. // J. Phys. Chem. 2015. V. 119. P. 29088.

[7] Кюркчан А.Г., Минаев С.А., Соловейчик А.Л. // Радиотехн. и электрон. 2001. Т. 46. № 6. С. 666; Kyurkchan A.G., Minaev S.A., Soloveichik A.L. // J. Commun. Technol. Electron. 2001. V. 46. P. 615.

[8] Anyutin A.P., Kyurkchan A.G., Manenkov S.A., Minaev S.A. // J. Quant. Spectrosc. Radiat. Transfer. 2006. V. 100. P. 26.

[9] Маненков С.A. // Радиотехника и электроника. 2018. Т. 63. № 1. C. 3; Manenkov S.A. // J. Commun. Technol. Electron. 2018. V. 63. N 1. P. 3.

[10] Кюркчан А.Г., Маненков С.А., Алероева Х.T. // Акустический журнал. 2014. Т. 60. № 1. С. 13; Kyurkchan A.G., Manenkov S.A., Aleroeva Kh.T. // Acoustical Physics. 2014. V. 60. N 1. P. 11.

[11] Kyurkchan A.G., Manenkov S.A. // J. Quant. Spectrosc. Radiat. Transfer. 2012. V. 113. P. 2368.

[12] Kyurkchan A.G, Manenkov S.A. // J. Quant. Spectrosc. Radiat. Transfer. 2018. V. 221. P. 243.

[13] Захаров Е.В., Пименов Ю.В. Численный анализ дифракции радиоволн. М.: Радио и связь, 1982. 184 с.

[14] Дмитриев В.И., Захаров Е.В. Интегральные уравнения в краевых задачах электродинамики. М.: Изд-во МГУ, 1987. $167 \mathrm{c}$. 
[15] Александров Д.В, Анютин А.П., Коршунов И.П, Шатров А.Д. // Изв. вузов. Радиофизика. 2017. Т. 60. № 2. C. 131; Aleksandrov D.V, Anyutin A.P., Korshunov I.P., Shatrov A.D. // Radiophysics and Quantum Electronics 2017. V. 60. N 2. P. 119.

[16] Анютин А.П., Кориунов И.П, Шатров А.Д. // Радиотехника и электроника. 2017. Т. 62. № 1. С. 35; Anyutin A.P., Korshunov I.P., Shatrov A.D. // J. Commun. Technol. Electron. 2017. V. 62. P. 31. 